\title{
THE ATOMIC DECOMPOSITION OF BESOV-BERGMAN-LIPSCHITZ SPACES
}

\author{
GERALDO SOARES DE SOUZA \\ Dedicated to Professor Daniel Waterman
}

ABSTRACT. Let $b$ denote a special atom, $b:[-\pi, \pi) \rightarrow R, b(t)=1 / 2 \pi$ or, for any interval $I$ in $[-\pi, \pi), b(t)=-|I|^{-1 / p} \chi_{R}(t)+|I|^{-1 / p} \chi_{L}(t), L$ is the left half of $I, R$ is the right half, $|I|$ denotes the length of $I$ and $\chi_{E}$ the characteristic function of $E$. For $1 / 2<p<\infty$, let $\left(b_{n}\right)$ be special atoms and $\left(c_{n}\right)$ a sequence of real numbers; then we define the space

$$
B^{p}=\left\{f:[-\pi, \pi) \rightarrow R ; f(t)=\sum_{n=1}^{\infty} c_{n} b_{n}(t), \sum_{n=1}^{\infty}\left|c_{n}\right|<\infty\right\} .
$$

We endow $B^{p}$ with the norm $\|f\|_{B^{p}}=\operatorname{Inf} \sum_{n=1}^{\infty}\left|c_{n}\right|$, where the infimum is taken over all possible representations of $f$.

In the early $1960 \mathrm{~s}$, the following spaces were introduced, now known as Besov-Bergman-Lipschitz spaces. For $0<\alpha<1,1 \leq r, s \leq \infty$, let

$$
\begin{aligned}
\Lambda(\alpha, r, s)=\{f:[ & -\pi, \pi) \rightarrow R,\|f\|_{\Lambda(\alpha, r, s)} \\
& \left.=\|f\|_{r}+\left(\int_{-\pi}^{\pi} \frac{\left(\|f(x+t)-f(x)\|_{r}\right)^{s}}{|t|^{1+\alpha s}} d t\right)^{1 / s}<\infty\right\}
\end{aligned}
$$

where \|\|$_{r}$ is the Lebesgue space $L^{r}$-norm.

Now we write down the main theorem of this paper which is as follows.

THEOREM C. $f \in B^{p}$ for $1<p<\infty$ if and only if $f \in \Lambda(1-1 / p, 1,1)$. Moreover, there are absolute constants $M$ and $N$ such that

$$
N\|f\|_{B^{p}} \leq\|f\|_{\Lambda(1-1 / p, 1,1)} \leq M\|f\|_{B^{p}} .
$$

Let $b$ denote a special atom, $b:[-\pi, \pi) \rightarrow R, b(t)=1 / 2 \pi$ or, for any interval $I$ in $[-\pi, \pi), b(t)=-|I|^{-1 / p} \chi_{R}(t)+|I|^{-1 / p} \chi_{L}(t), L$ is the left half of $I, R$ is the right half, $|I|$ denotes the length of $I$ and $\chi_{E}$ the characteristic function of $E$. For $1 / 2<p<\infty$, let $\left(b_{n}\right)$ be special atoms and $\left(c_{n}\right)$ a sequence of real numbers; then we define the space

$$
B^{p}=\left\{f:[-\pi, \pi) \rightarrow R ; f(t)=\sum_{n=1}^{\infty} c_{n} b_{n}(t), \sum_{n=1}^{\infty}\left|c_{n}\right|<\infty\right\} .
$$

We endow $B^{p}$ with the norm $\|f\|_{B^{p}}=\operatorname{Inf} \sum_{n=1}^{\infty}\left|c_{n}\right|$, where the infimum is taken over all possible representations of $f$.

These spaces were originally introduced by the author who has extensively studied them. The reader is referred to $[\mathbf{3}-\mathbf{1 1}]$.

Received by the editors August 30, 1984.

1980 Mathematics Subject Classification. Primary 42 A99.

Key words and phrases. Besov-Bergman-Lipschitz spaces, equivalence of Banach spaces, analytic functions, atomic decomposition. 
In the early 1960s the following spaces were introduced, now known as BesovBergman-Lipschitz spaces. For $0<\alpha<1,1 \leq r, s \leq \infty$, let

$$
\begin{aligned}
\Lambda(\alpha, r, s)=\{f:[-\pi, \pi) & \rightarrow R,\|f\|_{\Lambda(\alpha, r, s)} \\
& \left.=\|f\|_{r}+\left(\int_{-\pi}^{\pi} \frac{\left(\|f(x+t)-f(x)\|_{r}\right)^{s}}{|t|^{1+\alpha s}} d t\right)^{1 / s}<\infty\right\}
\end{aligned}
$$

where \|\|$_{r}$ is the Lebesgue space $L^{r}$-norm.

These spaces have been studied in depth in $[13,14,15$ and 17].

We have the following result:

Theorem A (EMbedding Theorem). If $f \in B^{p}, 1<p<\infty$, then $f \in$ $\Lambda(1-1 / p, 1,1)$. Moreover, $\|f\|_{\Lambda(1-1 / p, 1,1)} \leq C_{p}\|f\|_{B^{p}}$, where $C_{p}$ is an absolute constant depending only on $p$.

ProOF. First of all we notice that the operator $T_{a} f=f^{a}$, where $f^{a}(x)=f(x-a)$ maps $B^{p}$ into $B^{p}$ continuously, so that we just need to prove this result for

$$
f_{h}(t)=\frac{-1}{(2 h)^{1 / p}} \chi_{[-h, 0)}(t)+\frac{1}{(2 h)^{1 / p}} \chi_{[0, h]}(t), \quad h>0,
$$

which will follow easily from the estimate for

$$
g_{h}(t)=\frac{1}{h^{1 / p}} \chi_{[0, h]}(t) .
$$

Therefore, all we need to prove is that

$$
I_{p}=\int_{-\pi}^{\pi} \int_{-\pi}^{\pi} \frac{\left|g_{h}(x)-g_{h}(y)\right|}{|x-y|^{2-1 / p}} d x d y \leq C_{p}
$$

where $C_{p}$ depends only on $p$.

In fact,

$$
I_{p} \leq \frac{2}{h^{1 / p}} \int_{0}^{h} \int_{h}^{\infty} \frac{1}{|x-y|^{2-1 / p}} d x d y \leq \frac{2 p^{2}}{p-1} .
$$

Our argument shows that, for $f_{h}$ as above, we have $\left\|f_{h}\right\|_{\Lambda(1-1 / p, 1,1)}<C_{p}$, and consequently, if $f \in B^{p}$ it follows that $\|f\|_{\Lambda(1-1 / p, 1,1)} \leq C_{p}\|f\|_{B^{p}}$. So Theorem A is proved.

Because of Theorem $\mathrm{A}$ we may regard $B^{p}$ as a subset of $\Lambda(1-1 / p, 1,1)$, so that we have

LEMMA B. $B^{p}$ is a dense subset of $\Lambda(1-1 / p, 1,1)$.

Before we prove this lemma we would like to point out some definitions and results in order to make this presentation reasonably self-contained.

We define the Lipschitz space $\operatorname{Lip} \alpha=\{g:[-\pi, \pi) \rightarrow R$ continuous, such that $\left.g(x+h)-g(x)=O\left(h^{\alpha}\right)\right\}$ for $0<\alpha<1$. The infimum of the constants in the definition of $\operatorname{Lip} \alpha$ is taken as $\|g\|_{\operatorname{Lip} \alpha}$. Also $\operatorname{Lip}^{\prime} \alpha=\left\{g^{\prime}: g \in \operatorname{Lip} \alpha\right\}$, where the prime indicates differentiation. We endow $\operatorname{Lip}^{\prime} \alpha$ with the norm $\left\|g^{\prime}\right\|_{\mathrm{Lip}^{\prime} \alpha}=$ $\|g\|_{\text {Lipo }}$. 
A result that the interested reader can see in $[\mathbf{7}$ and 9$]$ is that the dual of $B^{p}$ is equivalent to $\operatorname{Lip}^{\prime} 1 / p$ for $1<p<\infty$. In the sense that if $\psi$ is a bounded linear functional on $B^{p}$ there is a unique $g$ in $\operatorname{Lip} 1 / p$ such that

$$
\psi(f)=\lim _{r \rightarrow 1} \int_{-\pi}^{\pi} f_{r}(t) g_{r}^{\prime}(t) d t \quad \text { for any } f \text { in } B^{p},
$$

where $g_{r}=P_{r} * g$ and $f_{r}=P_{r} * f$ is the Poisson integral of $g$ (respectively $f$ ).

In [13] Flett showed that the dual space of $\Lambda(1-1 / p, 1,1)$ is equivalent to $\operatorname{Lip}^{\prime} 1 / p$, by means of the same representation above.

PROOF OF LEMMA B. Clearly $B^{p}$ is closed under translations and dilations. If $B^{p}$ is not dense in $\Lambda(1-1 / p, 1,1)$, then there exist a $g \in \operatorname{Lip} 1 / p$ such that $\|g\|_{\text {Lip } 1 / p}=1$ and $\lim _{r \rightarrow 1} \int_{\pi}^{\pi} f_{r}(t) g_{r}^{\prime}(t) d t=0$, for any $f \in B^{p}$, where $g_{r}$ and $f_{r}$ are as above. But this must hold for any translation or dilation of $f$ in $B^{p}$. This implies $g$ is constant and $\|g\|_{\mathrm{Lip} 1 / p}=0$, which is absurd. Hence the lemma is proved.

Notice that we have the following situation, the spaces $B^{p}$ and $\Lambda(1-1 / p, 1,1)$ have the same duals; see comments right after Lemma B. Moreover Theorem A tells us that $B^{p}$ is regarded as a dense subset of $\Lambda(1-1 / p, 1,1)$, so that the classic theorem in functional analysis ensures us that $B^{p}$ and $\Lambda(1-1 / p, 1,1)$ are equivalent as Banach spaces; for instance see Theorem 4.14 of $[16$, p. 96] and Corollary $2.12 \mathrm{c}$ of $[16, \mathrm{p} .48]$. Really what we have proved is that the embedding operator $A: B^{p} \rightarrow \Lambda(1-1 / p, 1,1)$ defined by $A(f)=f$ is a Banach space isomorphism.

Now we write down the main theorem of this paper which is as follows:

THEOREM C. $f \in B^{p}$ for $1<p<\infty$ if and only if $f \in \Lambda(1-1 / p, 1,1)$. Moreover, there are absolute constants $M$ and $N$ such that

$$
N\|f\|_{B^{p}} \leq\|f\|_{\Lambda(1-1 / p, 1,1)} \leq M\|f\|_{B^{p}} .
$$

REMARK. The proof of Theorem A should neither require cancellation nor the special form of a special atom. This indicates that if we consider a function supported on an interval $I \subseteq[-\pi, \pi)$ and $|a(x)| \leq|I|^{-1 / p}$ then $a \in \Lambda(1-1 / p, 1,1)$ and $\|a\|_{\Lambda(1-1 / p, 1,1)} \leq M<\infty$ where $M$ is an absolute constant which depends only on $p$. Therefore, we can define the space

$$
A^{p}=\left\{f:[-\pi, \pi) \rightarrow R, f(t)=\sum_{n=1}^{\infty} c_{n} a_{n}(t), \sum_{n=1}^{\infty}\left|c_{n}\right|<\infty\right\},
$$

where $a_{k}$ is supported on an interval $I_{k}$ and $\left|a_{k}(t)\right| \leq\left|I_{k}\right|^{-1 / p}$. We endow $A^{p}$ with the norm $\|f\|_{A^{p}}=\operatorname{Inf} \sum_{n=1}^{\infty} c_{n} a_{n}(t)$, where the infimum is taken over all possible representations of $f$. Therefore, we have

$$
B^{p} \subseteq A^{p} \subseteq \Lambda(1-1 / p, 1,1)
$$

and consequently, $B^{p}$ is equivalent to $A^{p}$ as Banach spaces with equivalent norms.

We would like to point out that the proof that $A^{p}$ is continuously contained in $\Lambda(1-1 / p, 1,1)$ was done independently by Mitchell Taibleson [18] in a personal letter sent to me. Also in the same letter he pointed out that we could also have molecular representations of these spaces.

We recall that one of our earlier results done jointly with Richard O'Neil and G. Sampson (see $[\mathbf{9}]$ ) states that $B^{p}$ is equivalent with the spaces $J^{p}$ of all analytic 
functions in the disk for which

$$
\|F\|_{J^{p}}=|F(0)|+\frac{1}{\pi} \int_{0}^{1} \int_{-\pi}^{\pi}\left|F^{\prime}\left(r e^{i \theta}\right)\right|(1-r)^{1 / p-1} d \theta d r<\infty,
$$

in the sense that $f \in B^{p}$ if and only if $F \in J^{p}$; moreover, the norms are equivalents, and $\lim _{r \rightarrow 1} \operatorname{Re} F\left(r e^{i \theta}\right)=f(\theta)$ a.e., where the prime indicates differentiation.

The raison d'etre of this paper is that the equivalence of $B^{p}$ and $J^{p}$, as was done in [9], carries a very tedious calculation, which makes it very uncomfortable and hard to follow.

The above equivalence shows that $B^{p}$ can be identified with the boundary values of $J^{p}$, or we may regard it as an atomic decomposition of $J^{p}$, a real characterization.

This result, together with Theorem C, yields that $J^{p}$ is equivalent to $\Lambda(1-1 / p, 1,1)$, which was done by M. Tailbleson in $[17$, p. 421$]$.

For $p=1$ and other $p$ we refer the reader to $[4,5$ and 6$]$.

We believe that Theorem $\mathrm{C}$ will help us to have a better understanding of these Besov-Bergman-Lipschitz spaces.

Finally, we mention that the same technique used to prove Theorem $\mathrm{C}$ can be easily used to show that

$$
\int_{-\pi}^{\pi} \int_{-\pi}^{\pi} \frac{|f(x)-f(y)|}{|x-y|} d x d y<\infty \quad \text { for any } f \in B^{p} .
$$

A consequence of the boundedness of this integral is that any $f$ in $B^{p}$ satisfies Dini's condition and therefore the almost everywhere convergence is readily established (see $[\mathbf{1 1}]$ ).

We would like to thank Professor Mitchell Taibelson with whom we had several conversations about these spaces $B^{p}$.

\section{REFERENCES}

1. J. M. Anderson, J. Clunie and C. H. Pommerenke, On the Bloch functions and normal family, J. Reine Angew. Math. 270 (1974), 12-37.

2. O. V. Besov, On embedding and extension theorems for some function classes, Trudy Mat. Inst. Steklov. 60 (1960), 42-81.

3. G. S. de Souza, Spaces formed by special atoms, Ph.D. Dissertation, State Univ. New York at Albany, 1980.

4. Spaces formed with special atoms. II, Functional Analysis, Holomorphy and Approximation Theory. II, Elsevier Science Publ. B.V., North-Holland, Amsterdam, 1984.

5. __ Spaces formed by special atoms. I, Rocky Mountain J. Math. 14 (1984), 423-431.

6. G. S. de Souza and G. Sampson, A real characterization of the pre-dual of Bloch functions, J. London Math. Soc. (2) 27 (1983), 267-276.

7. _ Two theonems on interpolation of operators, J. Funct. Anal. 46 (1982), 149-157.

8. _ An interpolation of operators, An. Acad. Brasil. Ciênc. 54 (1982), 465-467.

9. G. S. de Souza, Richard O'Neil and G. Sampson, An analytic characterization of the special atoms spaces, Preprint 1983.

10. (Minneapolis, 1981), Lecture Notes in Math., vol. 908, Springer-Verlag, Berlin and New York, 1981.

11. _ On the convergence of Fourier series, Internat. J. Math. Math. Sci. (to appear).

12. P. Duren, The theory of $H^{p}$-spaces, Academic Press, New York, 1970.

13. T. M. Flett, On the rate of growth of the mean value of holomorphic and harmonic functions, Proc. London Math. Soc. (3) 20 (1970), 749-768. 
14. E. Stein, Singular integrals and differentiabritity properties of functions, Princeton Univ. Press, Princeton, N.J., 1970.

15. A. L. Shields and D. L. Williams, Bounded projections, duality and multipliers and spaces of analytic functions, Trans. Amer. Math. Soc. 162 (1971), 287-302.

16. Walter Rudin, Functional analysis, McGraw-Hill, New York, 1973.

17. M. Taibleson, On the theory of Lipschitz spaces and distributions on Euclidean $n$-space. I, II and III, J. Math. Mech. 13 (1964), 407-479; 14 (1965), 821-839; 15 (1966), 973-981.

18. , Personal communication.

19. A. Zygmund, Trigonometric series, Cambridge Univ. Press, London, 1959.

Department of Mathematics, Auburn University, Auburn, Alabama 36849 